\title{
Developmental Profile of Psychiatric Risk Associated With Voltage-Gated Cation Channel Activity
}

\author{
Nicholas E. Clifton, Leonardo Collado-Torres, Emily E. Burke, Antonio F. Pardiñas, \\ Janet C. Harwood, Arianna Di Florio, James T.R. Walters, Michael J. Owen, \\ Michael C. O’Donovan, Daniel R. Weinberger, Peter A. Holmans, Andrew E. Jaffe, and \\ Jeremy Hall
}

\begin{abstract}
BACKGROUND: Recent breakthroughs in psychiatric genetics have implicated biological pathways onto which genetic risk for psychiatric disorders converges. However, these studies do not reveal the developmental time point(s) at which these pathways are relevant.

METHODS: We aimed to determine the relationship between psychiatric risk and developmental gene expression relating to discrete biological pathways. We used postmortem RNA sequencing data (BrainSeq and BrainSpan) from brain tissue at multiple prenatal and postnatal time points, with summary statistics from recent genome-wide association studies of schizophrenia, bipolar disorder, and major depressive disorder. We prioritized gene sets for overall enrichment of association with each disorder and then tested the relationship between the association of their constituent genes with their relative expression at each developmental stage.

RESULTS: We observed relationships between the expression of genes involved in voltage-gated cation channel activity during early midfetal, adolescence, and early adulthood time points and association with schizophrenia and bipolar disorder, such that genes more strongly associated with these disorders had relatively low expression during early midfetal development and higher expression during adolescence and early adulthood. The relationship with schizophrenia was strongest for the subset of genes related to calcium channel activity, while for bipolar disorder, the relationship was distributed between calcium and potassium channel activity genes.

CONCLUSIONS: Our results indicate periods during development when biological pathways related to the activity of calcium and potassium channels may be most vulnerable to the effects of genetic variants conferring risk for psychiatric disorders. Furthermore, they indicate key time points and potential targets for disorder-specific therapeutic interventions.
\end{abstract}

https://doi.org/10.1016/j.biopsych.2021.03.009

Biological insight to psychiatric disorders has come from the identification of molecular pathways enriched for genetic association, determined by large cohort genome-wide studies. However, the expression of many genes varies as a function of age, and therefore, the relevance of genetically associated pathways likely varies across development.

Schizophrenia, bipolar disorder (BD), and major depressive disorder (MDD) show considerable heritability and share a substantial component of genetic risk (1-6). Variance between the disorders may in part be attributed to differences in the degree of neurodevelopmental impairment $(3,7,8)$. Many psychiatric symptoms first present during adolescence or in early adulthood (9), implying that pathophysiology emerges as the brain matures (10). However, there is substantial evidence that altered neurodevelopment during earlier prenatal or postnatal periods may contribute to some, if not all, psychiatric disorders $(3,11-14)$. Identifying the developmental stage at which particular biological pathways are most likely to contribute to risk for psychiatric disorders is therefore an important step toward understanding disease etiology and targeting new treatments.

Genetic association studies of schizophrenia have consistently demonstrated a convergence of genetic risk upon sets of genes with synaptic functions (15-19), including discrete signal transduction pathways such as voltage-gated calcium channel complexes and glutamate receptor complexes. The most recent genome-wide association studies (GWASs) of BD and MDD also highlight genes and pathways related to synaptic activity (20-23). Hence, signaling complexes in the synaptic membrane represent strong candidates for psychiatric drug targeting. 
Neurodevelopment is regulated by a program of tightly controlled gene expression $(24,25)$. The majority of GWAS loci linked to psychiatric disorders are noncoding and most likely mediate risk by affecting gene expression (26-29). Genes harboring risk for schizophrenia and BD are also among those most dynamically expressed across development, particularly in prefrontal cortical regions (30-32). However, the question of the developmental stage at which genes and gene pathways associated with major psychiatric diseases are primarily expressed remains largely unresolved, limiting our ability to reduce risk and target treatments.

By integrating developmental transcriptomic data of the human brain with GWAS data of genetic risk in schizophrenia, $B D$, and MDD, we aimed to identify time points at which gene sets implicated in risk for psychiatric disorders are most strongly expressed, in view of highlighting periods when key biological pathways may preferentially confer risk to these disorders.

\section{METHODS AND MATERIALS}

\section{Genotype Data}

We obtained common variant summary statistics from published GWAS data. The schizophrenia sample $(40,675$ cases, 64,643 controls) (17) is a meta-analysis of a GWAS derived from UK cohorts of patients taking clozapine $(11,260$ cases, 24,542 controls) and an international Psychiatric Genomics Consortium (PGC) study (29,415 cases, 40,101 controls) (33). Case-control samples for BD were compiled by the PGC from 32 cohorts of European descent $(20,352$ cases, 31,358 controls) (21). Genotype data for MDD (135,458 cases, 344,901 controls) were derived from a PGC meta-analysis of 7 cohorts of European descent (22). For direct comparison of genetic factors contributing to schizophrenia and BD, we utilized additional summary statistics from a published GWAS of cases (23,585 schizophrenia, 15,270 BD) matched for ancestry and genotyping platform (34).

\section{Transcriptomic Data}

Human brain transcriptomic data from across the life span were obtained from two sources. A primary dataset was derived from postmortem dorsolateral prefrontal cortex (DLPFC) of 336 individuals with no history of psychiatric condition, referred to as BrainSeq (31). Samples ranged in age from second trimester to 85 years (Table S1 in Supplement 2). Tissue acquisition, processing, and genotyping have been described previously $(31,35)$. We obtained a second, smaller human developmental transcriptomic dataset from the Allen Institute BrainSpan Atlas $(36,37)$, from which we retained data for DLPFC samples $(n=40)$ ranging from the first trimester to 40 years (Table S1 in Supplement 2). Sixteen samples from Brainseq and BrainSpan originated from the same individuals; these were removed from Brainseq to ensure independence. Raw sequencing reads from BrainSeq and BrainSpan were processed using the same software pipeline (38-41). Gene counts were converted to reads per kilobase of transcript per million mapped reads (RPKM) values (calculated in relation to the number of gene-assigned reads).

\section{Gene Sets}

Gene sets defined by biological pathways were curated from the Gene Ontology (GO) database (42) (downloaded January $13,2020)$, excluding gene annotations with evidence codes NAS (non-traceable author statement), IEA (inferred from electronic annotation), or RCA (inferred from reviewed computational analysis). For primary analyses, gene sets containing fewer than 100 genes were excluded to minimize the effect of outliers. After filtering, 1766 gene sets remained (Table S2A in Supplement 2).

Further GO definitions were obtained from the SynGO database (43), consisting of manually curated gene annotations of synaptic function and location. After filtering for sets containing at least 100 genes, 19 sets remained (Table S4A in Supplement 2).

\section{Gene Set Association Analysis}

Gene set association analysis was performed in MAGMA (version 1.08b) (44). Using GWAS summary statistics, single nucleotide polymorphisms with a minor allele frequency greater than $1 \%$ were annotated to genes, allowing for proximal regulatory regions (35 kb upstream, $10 \mathrm{~kb}$ downstream) (45). Gene-wide association statistics were calculated using the single nucleotide polymorphism-wise mean model with the 1000 Genomes European reference panel (46) to control for linkage disequilibrium. Gene set association was calculated using a background of all genes, conditional on the brain expression of each gene. Brain expression was defined as $\log _{2}(\mathrm{RPKM}+0.5)$, where RPKM is the mean average across BrainSeq samples.

Primary analyses aimed to identify gene sets significantly enriched for GWAS association signal. Stepwise conditional analyses were applied to significantly (false discovery rate $[F D R]<0.05)(47)$ associated gene sets to identify a reduced number of independent sets that efficiently summarize the biological themes underlying the association. During this process, gene sets were repeatedly retested for genetic association, each time selecting the set with the highest effect size and adding that set to the conditional variables. On each iteration, gene sets that were no longer nominally significant (unadjusted $p \geq .05$ ) were excluded. This procedure resulted in gene sets that may have small amounts of overlap but contribute substantially distinct genetic risk.

In secondary analyses, we tested the relative association of subsets compared with a larger set by adding the larger set to the model as a conditional variable. Comparisons of genetic association between nonoverlapping gene sets were performed using a $z$ test of beta values.

\section{Developmental Gene Expression Scores}

Transcriptomic data were divided into developmental stages (Table S1 in Supplement 2). Stages containing fewer than two samples in BrainSpan or BrainSeq were excluded for that dataset. Accordingly, two samples from BrainSeq were excluded (one early fetal, one late infancy). Late adulthood was not represented in BrainSpan. To permit comparison between genes and control for covariates, we constructed a score measuring the expression of each gene at each developmental stage relative to all other stages (30). Expression scores were 
calculated by fitting a linear regression model to each gene using limma (48) and extracting the $t$ statistic for each developmental stage term:

$$
\text { Expression } \sim \sum_{i=1}^{5} \operatorname{snpPC}_{i}+\text { sex }+ \text { developmental stage }
$$

Developmental stage is a binary variable indicating whether the sample is from that stage or not. We controlled for genetic ancestry by covarying for the first five principal components defining sample genotypes (snpPC).

\section{Expression-Association Relationships}

For selected gene sets, we assessed the relationship between developmental stage gene expression and association with a disorder using MAGMA (version 1.08b) interaction analysis (49):

$$
Z=\beta_{0}+B \beta_{B}+S_{1} \beta_{1}+S_{2} \beta_{2}+S_{12} \beta_{12}+\varepsilon
$$

In this model, the interaction term $S_{12}$ is defined as the product of gene set membership $S_{1}$ and gene property $S_{2}$ terms. This method determined whether enrichment for genetic association in a gene set is stronger for genes with a higher expression score. Because of sensitivity to outlier effects in smaller sets, primary analyses were restricted to sets $\geq 100$ genes (49). Interaction analyses were two-tailed, and statistical correction was performed for each gene set at each developmental stage using the Bonferroni method (p.adj). Analyses yielding significant interactions in one disorder were repeated as secondary analyses in the remaining disorders.

\section{Clustering of Developmental Expression Trajectories}

To generalize expression analyses specific to each developmental stage to the complete expression trajectory across all stages, we partitioned gene sets of interest into subsets of genes with similar trajectories. Gene expression at each stage (mean RPKM) was scaled by subtracting the mean and dividing by the standard deviation. A suitable number of clusters for K-means clustering was identified using a scree plot. The procedure was run using 20 sets of random partitions, allowing for a maximum of 20 iterations. Gene set association analyses were used to test for enrichment of GWAS signal in gene clusters.

\section{RESULTS}

\section{Independently Associated Gene Ontologies}

To select gene sets for downstream analyses, we performed gene set association analysis on a filtered list of $1766 \mathrm{GO}-$ derived gene sets. After FDR correction, we observed 57 gene sets enriched for association with schizophrenia (Table S2A in Supplement 2) and 11 associated with BD (Table S2B in Supplement 2). No sets remained significantly associated with MDD (Table S2C in Supplement 2). Because of redundancy between GO terms, we used conditional analyses to select gene sets with independent associations. This procedure yielded six sets independently associated with schizophrenia (Table S2A in Supplement 2) and two sets independently associated with BD (Table S2B in Supplement 2). Genes related to voltage-gated cation channel activity
(VG-cation) were enriched for association with schizophrenia $\left(\beta=.43, \mathrm{FDR}=6.2 \times 10^{-4}\right)$ and $\mathrm{BD}(\beta=.32, \mathrm{FDR}=0.025)$, but not MDD $(\beta=.14$, FDR =1.0). Of the 125 genes in the VGcation set, only 1 gene, CACNA1C, surpassed gene-wide significance $\left(p<2.5 \times 10^{-6}\right)(50)$ for association with both schizophrenia and BD (Figure S1A in Supplement 1). Using a more exploratory threshold of gene-wide association $(p<1 \times$ $10^{-4}$ ), we noted three further genes contributing risk to both disorders (Figure S1B-D in Supplement 1).

\section{Developmental Stage-Specific Relationships Between Gene Expression and Genetic Association Within Biological Pathways}

We used BrainSeq transcriptomic data to determine whether enrichment for common variant association in gene sets with significant independent main effects is stronger for genes preferentially expressed during particular developmental stages. In analyses of genes annotated by VG-cation, we observed a significant positive interaction term for the relationship between expression during early adulthood and genetic association with both schizophrenia $(\beta=.15$, p.adj $=.0090)($ Table S3A in Supplement 2$)$ and BD $(\beta=.15$, p.adj = .0022) (Table S3B in Supplement 2; Figure 1A, B). This indicates that during early adulthood, VG-cation genes more strongly associated with schizophrenia or BD have relatively high DLPFC expression than during other developmental stages, while those lacking association have relatively low expression (Figure S2 in Supplement 1). Conversely, during early midfetal development (10-15 postconceptual weeks), there was a negative relationship (indicating that genes more associated with the disorder have relatively low DLPFC expression and less associated genes have higher expression) between VG-cation expression and association with BD $(\beta=-.047, p$. adj $=.011)$.

For comparison, we tested these temporal relationships with VG-cation expression in MDD genetic risk. VG-cation expression was not related to association with MDD at any developmental stage (Figure 1C).

We repeated analyses of developmental VG-cation expression using an independent transcriptomic dataset, BrainSpan. Overall, the pattern of preferential VG-cation expression across development was consistent between BrainSeq and BrainSpan (Figure 1D-F), such that early embryonic expression exhibited the strongest negative relationship with association with schizophrenia (early fetal: $\beta=-.16$, $p=.0035$; early midfetal: $\beta=-.053, p=.30$ ) and BD (early fetal: $\beta=-.084, p=.086$; early midfetal: $\beta=-.10, p=.024)$, and positive relationships were observed during early adulthood (schizophrenia: $\beta=.32, p=.0017$; BD: $\beta=.26, p=.0071$ ). BrainSpan VG-cation gene expression during adolescence was also strongly positively related to association with schizophrenia $\left(\beta=.32, p=8.7 \times 10^{-5}\right)$ and $\operatorname{BD}(\beta=.21, p=$ .0038). Again, no relationships were observed between VGcation temporal expression and MDD association.

Multiple synaptic pathways, including those related to voltage-gated ion channels, have been previously implicated in risk for psychiatric disorders (15). To assess the specificity of our findings, we studied an independent dataset of synaptic gene annotations for generalization. Using manually curated 

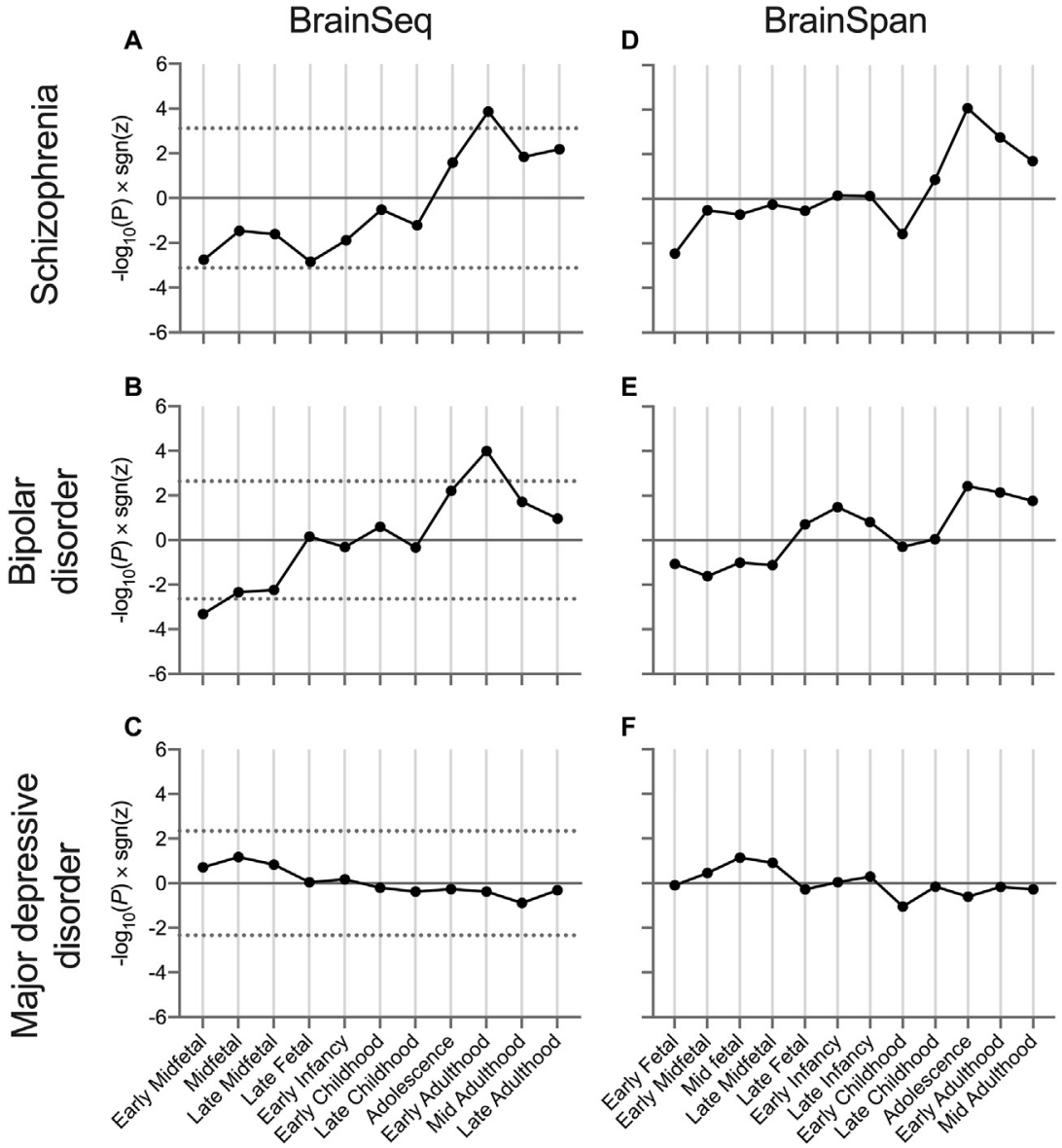

Figure 1. Developmental stage-specific relationships between the expression of voltage-gated cation channel activity genes and genetic association with psychiatric disorders. Shown is $-\log _{10}(P) \times \operatorname{sgn}(z)$ from independent MAGMA interaction analyses of common variant association and gene expression scores derived from BrainSeq (A-C) or BrainSpan (D-F) at each developmental stage, compared with a background of all genes. Dotted lines indicate thresholds for statistical significance after correcting for analyses of all gene sets with independent association with the disorder and all stages (Table S3A, B in Supplement 2). Major depressive disorder analyses were corrected for the number of stages only. Developmental stages containing fewer than two samples in the dataset are not represented. early fetal, 8-9 pcw; early midfetal, 10-15 pcw; midfetal, $16 \mathrm{pcw}$; late midfetal, $17-23 \mathrm{pcw}$; late fetal, $24-37 \mathrm{pcw}$; early infancy, 0-5 months; late infancy, 6-11 months; early childhood, 1-5 years; late childhood, 6-12 years; adolescence, 13-19 years; early adulthood, 20-29 years; mid adulthood, 30-59 years; late adulthood, 60-100 years. pcw, postconceptual weeks. gene sets from the SynGO database (43), we repeated gene set association and developmental interaction analyses. From these sets, independent genetic associations were found for three gene sets with schizophrenia and three with BD (Table S4A, B in Supplement 2). None of these SynGO gene sets exhibited developmental patterns of expression that were linked to genetic association (Table S5A, B in Supplement 2).

\section{Partitioning Association Between Child Terms of Voltage-Gated Cation Channel Activity}

The VG-cation term (125 genes) is composed of four nested "child" GO terms: voltage-gated potassium channel activity (VG-potassium, 80 genes), voltage-gated calcium channel activity (VG-calcium, 38 genes), NMDA glutamate receptor activity ( 7 genes), and voltage-gated proton channel activity (1 gene). To examine whether genetic association of VG-cation with schizophrenia and BD is further enriched in the smaller and more biologically specific "child" terms (excluding "voltage-gated proton channel activity" owing to size), we performed gene set analysis of these terms conditional on VGcation. VG-calcium showed greater enrichment for association with schizophrenia than the full VG-cation set (Table 1). Conversely, association with BD was not further enriched within the "child" terms (Table 1), indicating even distribution across subtypes of VG-cation.

Based on the differing contributions of VG-cation "child" terms to genetic association with schizophrenia and BD, we hypothesized that VG-calcium and VG-potassium are points of genetic divergence between the two disorders. We repeated the above gene set analyses using summary statistics from a schizophrenia versus BD GWAS (34). We found differential enrichment for genetic association with schizophrenia and $\mathrm{BD}$ in VG-cation genes $(\beta=.19, p=.0084)$, which manifested significantly in VG-potassium $(\beta=.21, p=.015)$ but not VGcalcium $(\beta=.15, p=.16)$ (Table 1).

We examined the contribution of VG-calcium and VGpotassium to the developmental patterns of preferential risk gene expression in VG-cation. Developmental relationships during early midfetal and early adulthood stages between gene expression and schizophrenia association were more prominent in VG-calcium than in VG-potassium genes (early midfetal: $z$ test $p=.039$; early adulthood: $z$ test $p=.020$ ) (Figure 2A). Conversely, these relationships in analyses of $\mathrm{BD}$ appeared more pronounced among VG-potassium genes (Figure 2B), although comparisons of effect sizes showed no significant differences at these stages (early midfetal: $z$ test $p=.49$; early adulthood: $z$ test $p=.20$ ), likely owing to the larger set size of VG-potassium than 
Table 1. The Relative Genetic Association of Voltage-Gated Cation Channel Activity "Child" Terms Compared With the Complete Gene Set in Two Psychiatric Disorders

\begin{tabular}{|c|c|c|c|c|c|c|c|c|c|}
\hline \multirow[b]{2}{*}{ Gene Set } & \multirow[b]{2}{*}{$n$} & \multicolumn{3}{|c|}{ Schizophrenia } & \multicolumn{3}{|c|}{ Bipolar Disorder } & \multicolumn{2}{|c|}{$\begin{array}{l}\text { Schizophrenia } \\
\text { vs. Bipolar } \\
\text { Disorder }\end{array}$} \\
\hline & & $\beta$ & $p$ Value & $\begin{array}{l}\text { p.conditional } \\
\text { Value }\end{array}$ & $\beta$ & $p$ Value & $\begin{array}{l}\text { p.conditional } \\
\text { Value }\end{array}$ & $\beta$ & $p$ Value \\
\hline $\begin{array}{l}\text { Voltage-Gated Cation } \\
\text { Channel Activity }\end{array}$ & 125 & .43 & $2.0 \times 10^{-6}$ & NA & .32 & $9.8 \times 10^{-5}$ & NA & .19 & .0084 \\
\hline $\begin{array}{l}\text { Voltage-Gated Calcium } \\
\text { Channel Activity }\end{array}$ & 38 & .98 & $3.7 \times 10^{-9}$ & $4.8 \times 10^{-5}$ & .41 & .0064 & .26 & .15 & .16 \\
\hline $\begin{array}{l}\text { Voltage-Gated } \\
\text { Potassium } \\
\text { Channel Activity }\end{array}$ & 80 & .12 & .15 & 1.0 & .25 & .0080 & .87 & .21 & .015 \\
\hline $\begin{array}{l}\text { NMDA Glutamate } \\
\text { Receptor Activity }\end{array}$ & 7 & .90 & .014 & .12 & .61 & .046 & .20 & .12 & .35 \\
\hline
\end{tabular}

Shown is the output from gene set association analysis in MAGMA before ( $p$ value) and after ( $p$.conditional value) conditioning on the full voltagegated cation channel activity gene set. Also shown are results from contrasting the genetic contributions to the two disorders by gene set association analyses with summary statistics from schizophrenia vs. bipolar disorder genome-wide association study.

NA, not applicable.

VG-calcium, giving greater significance for a given effect size. These analyses also strengthened the evidence that positive expression-association relationships begin in adolescence and continue into early adulthood.

Together, these results suggest that the VG-cation subset involved in calcium activity are further enriched for association with schizophrenia, with those more strongly associated being preferentially expressed during adolescence and early adulthood. In contrast, calcium and potassium subsets exhibit a more balanced association with BD and a greater dominance of preferential risk gene expression during early adulthood among potassium activity genes.

\section{Partitioning Association by Developmental Expression Trajectory}

Our developmental stage-specific analyses suggest that VGcation genes with low early midfetal and high adolescence/ early adulthood expression are enriched for association with schizophrenia and BD. They also suggest that the schizophrenia association is stronger for the VG-calcium subset. To generalize these hypotheses across all developmental stages, we identified subsets of genes with similar expression trajectories and tested the distribution of genetic association. K- means clustering identified four broad expression trajectories in VG-cation genes (Figure 3; Figure S3 in Supplement 1; Table S6 in Supplement 2). Association with schizophrenia and $\mathrm{BD}$ was restricted to clusters 1 and 2, both of which contain genes with lower embryonic expression and higher expression in later life (Figure 3). Only cluster 2 (containing genes exhibiting later peak expression than cluster 1) harbored greater enrichment for association with schizophrenia and BD than VG-cation genes as a whole (schizophrenia: $p$.conditional = $3.6 \times 10^{-5} ; \mathrm{BD}$ : $p$.conditional $=.010$ ).

On splitting these clusters into VG-calcium and VGpotassium, we observed that the association of clusters 1 and 2 with schizophrenia was stronger among VG-calcium genes than among VG-potassium genes (cluster $1: z$ test $p=$ .0019 ; cluster 2 : $z$ test $p=6.4 \times 10^{-4}$ ), and only the VGcalcium subsets showed greater enrichment for association than VG-cation as a whole (VG-calcium cluster 1: p.conditional $=.0062 ;$ VG-calcium cluster 2 : p.conditional $=2.6 \times$ $10^{-6}$; VG-potassium cluster 1: p.conditional = .99; VGpotassium cluster 2: $p$.conditional $=.36$ ). Association of the same clusters with BD was distributed between VG-calcium and VG-potassium subsets (cluster $1: z$ test $p=.98$; cluster 2: $z$ test $p=.75$ ), which were not more enriched than the full

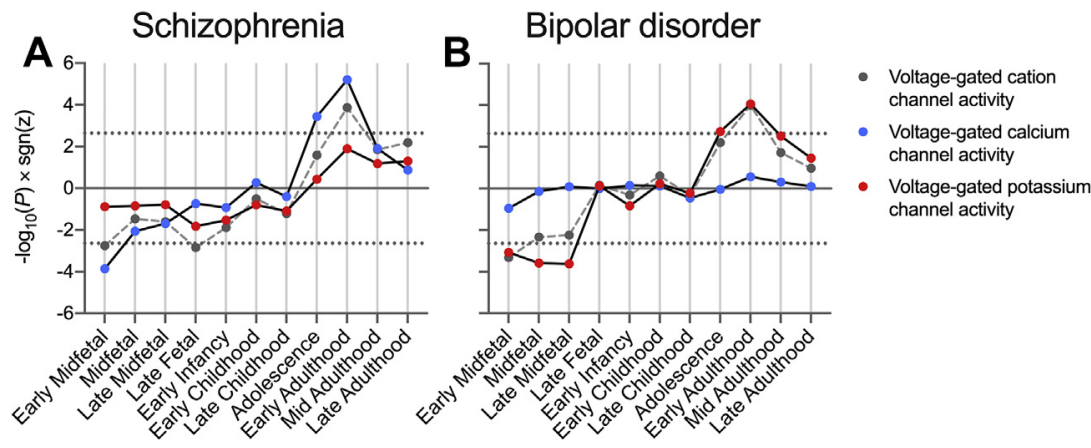

Figure 2. Comparison of expression-association relationships at 11 developmental stages within voltage-gated cation channel activity "child" terms. Shown is $-\log _{10}(P) \times \operatorname{sgn}(z)$ from MAGMA interaction analyses of schizophrenia (A) or bipolar disorder (B) common variant association and BrainSeq-derived gene expression scores at each stage, compared with a background of all genes. Dotted lines indicate thresholds for statistical significance after correcting for analyses of two gene sets across 11 stages. GO:0022843 voltage-gated cation channel activity, $n=125$; GO:0005245 voltage-gated calcium channel activity, $n=38$; GO:0005249 voltage-gated potassium channel activity, $n=80$. 

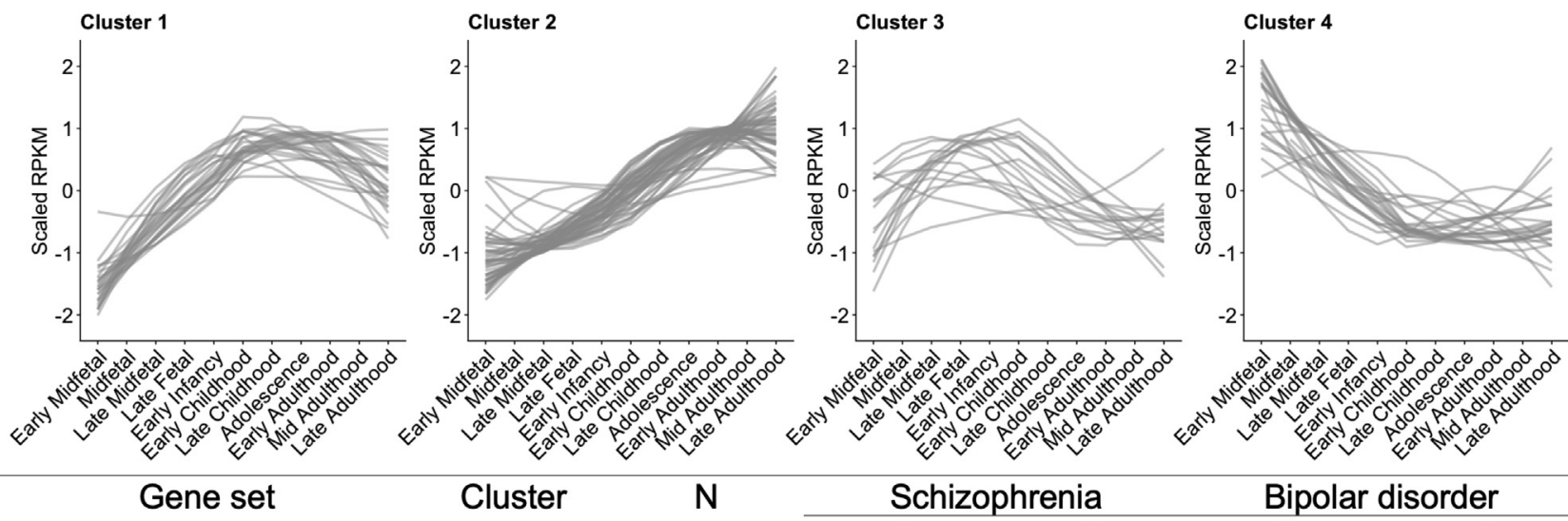

\section{Voltage-gated cation channel activity}

\begin{tabular}{ll}
1 & 27 \\
2 & 5 \\
3 & 17 \\
\hline 4 & 25
\end{tabular}

27

55

17

Voltage-gated calcium channel activity

\begin{tabular}{rc}
1 & 9 \\
\hline 2 & 1 \\
\hline 3 & 3 \\
\hline 4 & 1
\end{tabular}

25
9

9

\begin{tabular}{ll}
1 & 17 \\
2 & 36 \\
3 & 11 \\
\hline 4 & 15
\end{tabular}

3
10
17

Voltage-gated potassium channel activity

$\begin{array}{r}1 \\ 2 \\ \hline 3\end{array}$

17

36

15

$\beta$

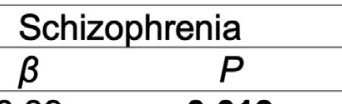

Bipolar disorder

\begin{tabular}{cccc}
$\beta$ & $P$ & $\beta$ & $P$ \\
0.39 & $\mathbf{0 . 0 1 9}$ & 0.36 & $\mathbf{0 . 0 2 3}$ \\
0.85 & $\mathbf{6 . 9 \times 1 0 ^ { - 1 0 }}$ & 0.52 & $\mathbf{1 . 4 \times 1 0 ^ { - 0 5 }}$ \\
-0.09 & 0.62 & 0.39 & 0.084 \\
\hline-0.14 & 0.75 & -0.26 & 0.92 \\
1.19 & $\mathbf{8 . 4 \times 1 0 ^ { - 0 5 }}$ & 0.38 & 0.14 \\
1.59 & $\mathbf{3 . 0 \times 1 0 ^ { - 0 9 }}$ & 0.58 & $\mathbf{0 . 0 0 8 5}$ \\
0.48 & 0.20 & 0.51 & 0.19 \\
-0.09 & 0.61 & 0.081 & 0.40 \\
-0.04 & 0.57 & 0.37 & $\mathbf{0 . 0 4 5}$ \\
0.49 & $\mathbf{0 . 0 0 2 1}$ & 0.49 & $\mathbf{6 . 2 \times 1 0 ^ { - 0 4 }}$ \\
-0.54 & 0.92 & 0.35 & 0.17 \\
-0.23 & 0.81 & -0.54 & 0.99
\end{tabular}

Figure 3. Clusters of developmental expression trajectories of voltage-gated cation channel activity genes and their enrichments for genome-wide association study association in schizophrenia and bipolar disorder. K-means clustering was used to divide genes based on their mean RPKM expression in BrainSeq across 11 developmental stages. Tabulated are results from schizophrenia and bipolar disorder gene set association analyses of each expression trajectory cluster, with separation into voltage-gated calcium channel activity and voltage-gated potassium channel activity "child" terms. RPKM, reads per kilobase of transcript per million mapped reads.

VG-cation gene set (VG-calcium cluster 1: p.conditional = .42; VG-calcium cluster 2: p.conditional = .11; VG-potassium cluster 1: $p$.conditional $=.39$; VG-potassium cluster 2 : $p$.conditional $=.081)$.

\section{DISCUSSION}

Our study highlights biological convergence among common variants conferring risk of schizophrenia and BD in genes annotated by the ontological term "voltage-gated cation channel activity" (VG-cation) and identifies adolescence and early adulthood as periods of preferential expression for the most strongly associated members in both disorders. In schizophrenia, these temporal relationships derived predominantly from the calcium-related component of VG-cation, VGcalcium, whereas in $\mathrm{BD}$, they were driven by both VG-calcium and VG-potassium components. Conversely, we observed no relationship between genetic association with MDD and preferential VG-cation gene expression at any developmental stage.

Enrichment for genetic association with schizophrenia, $\mathrm{BD}$, and MDD in genes encoding voltage-gated calcium channel subunits has been consistently reported in primary
GWAS $(17,21,22)$, with additional genetic evidence supporting their involvement in schizophrenia from rare variants $(19,51)$. While molecular abnormalities in calcium activity have historically been regarded as specific to BD (52-54), recent work suggests that alterations in cellular calcium may be comparable between schizophrenia and $\operatorname{BD}(55,56)$. The voltage-gated calcium channel family are signal transducers of electrical excitation in neurons. Through the induction of intracellular calcium transients, they couple membrane depolarization to signaling cascades including activitydependent regulation of gene expression (57). In this way, voltage-gated calcium channels influence synaptic plasticity and are thought to be important drivers of learning and memory (58).

Unlike schizophrenia, genetic association with BD in VGcation was distributed between calcium and potassium components. It is typically unfeasible to compare across disorders owing to differences in statistical power; however, we used summary statistics from a recent schizophrenia versus BD GWAS (34) to demonstrate that VG-potassium genes are differentially enriched for association with schizophrenia and BD. Voltage-gated potassium channels have been linked to BD through genetic studies previously 
(21,59-63), and this represents a divergence from schizophrenia and MDD, which lack evidence of conferring risk through potassium channels. Notably, the GO term "response to potassium ion" was the only pathway reported to be significantly differentially enriched for association in gene set analyses from the primary schizophrenia versus BD GWAS (34). The broad function of voltage-gated potassium channels is to control the threshold for action potentials and repolarize the membrane after firing (64). Similar to calcium channels, they have been shown to contribute to synaptic plasticity $(65,66)$.

Our results suggest that adolescence and early adulthood are periods of peak preferential DLPFC expression of VGcation genes associated with schizophrenia and BD. This lends to the idea that these developmental stages, which correspond to peak periods of symptom onset, may be more vulnerable to risk conferred through cation channels. Conversely, early midfetal development was characterized by comparably low expression of risk VG-cation genes, perhaps indicating a period of low vulnerability in this pathway. These conclusions are drawn from the assumption that peak expression relates to periods of high functional activity and the greatest impact of genetic variants. We cannot rule out, however, that change in the function of a gene expressed in relatively low abundance could mediate substantial risk.

More specifically, these periods of preferential gene expression in schizophrenia-associated VG-cation genes were characterized by more prominent relationships within the VG-calcium subset. Conversely, preferential expression of BD-associated VG-cation genes was agnostic to the cation subset. This discrepancy raises the possibility that VG-calcium genes, but not VG-potassium genes, contribute to an adolescence/early adulthood vulnerability to schizophrenia, while both subsets contribute to BD vulnerability during the same period.

Because of research bias and varying evidence quality, the database of GO annotations is undoubtedly incomplete. For example, the VG-cation GO term examined here does not include subsets related to sodium channel activity. Voltagegated sodium channels have been linked to schizophrenia and autism in sequencing studies $(51,67,68)$ and are involved in the pharmacodynamics of mood stabilizers used in the treatment of BD (69). Second, VG-calcium includes a small number of stargazin-like proteins, initially linked to voltagegated calcium channels via homology, but with functions related to membrane expression of AMPA receptors, and are now collectively known as transmembrane AMPA receptor regulatory proteins (70).

Because of its manual curation, the SynGO database of synaptic annotations (43) is likely to have good functional accuracy. Although these data do not contain annotations of VGcation genes, they include multiple gene sets annotated to synaptic pathways consistently implicated in psychiatric disorders (15-19). While some SynGO terms were enriched for association with schizophrenia or $\mathrm{BD}$, the risk conferred was not related to periods of preferential gene expression. These results provide evidence that the developmental profile of schizophrenia and BD risk associated with GO VG-cation genes does not generalize to other synaptic pathways.
Our results reflect trends in gene sets, but expression patterns of individual genes or transcripts associated with psychiatric disorder may vary. For example, CACNA1C (encoding calcium channel Cav1.2) contains a locus with genome-wide evidence for association with schizophrenia, BD, and MDD $(17,21,71-73)$ and is the single-gene cause of Timothy syndrome, a disorder of multiorgan maldevelopment (74). CACNA1C is reported to reach peak brain expression in late fetal/early childhood development $(75,76)$ and its forebrain deletion in embryonic, but not adult, mice models endophenotypes of psychiatric disorders (77). However, around 250 splice variants of CACNA1C have been reported (78), with varying expression profiles. Furthermore, it is still unclear which of the CACNA1C transcripts is associated with risk variants identified from GWAS. To better understand developmental vulnerability to genetic risk conferred through cation channels, the expression patterns of transcripts relevant to psychiatric disorders should be characterized independently of the gene-wide expression.

Our study employed transcriptomic data derived from the DLPFC, a region long thought to be affected in schizophrenia and mood disorders (79-81). Compared with other brain regions, the DLPFC is considered to be late maturing $(82,83)$ and exhibits some distinct developmental gene expression trajectories $(84,85)$. Hence, the relationships between genetic risk and developmental expression in other brain regions might be different or create age-specific vulnerabilities in other biological pathways that could account for the emergence of different psychiatric symptoms.

There is strong evidence that early developmental stages contribute to psychiatric pathophysiology, yet manifest as a disorder later in the life as the brain matures $(11,80)$. Using similar methodology, our previous work (30) demonstrated that among all brain-expressed genes, there is a bias for those with stronger genetic association to be preferentially expressed in the prefrontal cortex during early midfetal development (schizophrenia) or early infancy (schizophrenia/BD). Other biological pathways associated with psychiatric disorders may be most vulnerable to genetic risk during prenatal or early postnatal life, but were not identified by this study.

Our findings have implications for the targeting of therapeutics. Psychiatric disorders are insufficiently treated, yet voltage-gated cation channels have been recognized as potential targets for new (or existing) compounds $(66,86)$, and their druggability is an area of active research $(87,88)$. Preferential expression of genetically associated genes during ages typical of diagnosis reinforces the suitability of voltage-gated cation channels as targets. Our results also indicate periods when therapeutic agents acting on such pathways may be most effective.

\section{ACKNOWLEDGMENTS AND DISCLOSURES}

This study was supported by the Medical Research Council (Grant Nos. MR/ L010305/1 [to MJO] and MR/R011397/1 [to JH]) and a Wellcome Trust (204824/Z/16/Z [to NEC] and 100202/Z/12/Z [to MJO, JH]).

We thank the Bipolar Disorder and Major Depressive Disorder workgroups of the Psychiatric Genomics Consortium for providing summary statistics used in this study. We would like to thank the research participants and employees of 23andMe for making this work possible. We thank R. 
Zielke, R.D. Vigorito, and R.M. Johnson of the National Institute of Child Health and Human Development Brain and Tissue Bank for Developmental Disorders at the University of Maryland for providing fetal, child, and adolescent brain specimens. We also acknowledge the following brain bank collections: the Mount Sinai NIH Brain and Tissue Repository, the University of Pennsylvania Alzheimer's Disease Core Center, the University of Pittsburgh NeuroBioBank and Brain and Tissue Repositories, and the National Institute of Mental Health (NIMH) Human Brain Collection Core. Lastly, we thank the CommonMind Consortium: Pamela Sklar and Joseph Buxbaum (Icahn School of Medicine at Mount Sinai), Bernie Devlin and David Lewis (University of Pittsburgh), Raquel Gur and Chang-Gyu Hahn (University of Pennsylvania), Keisuke Hirai and Hiroyoshi Toyoshiba (Takeda Pharmaceuticals Company Limited), Enrico Domenici and Laurent Essioux (F. Hoffman-La Roche Ltd), Lara Mangravite and Mette Peters (Sage Bionetworks), and Thomas Lehner and Barbara Lipska (NIMH).

A previous version of this article was published as a preprint on bioRxiv: https://doi.org/10.1101/2020.10.19.345801.

The authors report no biomedical financial interests or potential conflicts of interest.

\section{ARTICLE INFORMATION}

From the Neuroscience and Mental Health Research Institute (NEC, MJO, $\mathrm{JH})$, Cardiff University; MRC Centre for Neuropsychiatric Genetics and Genomics (NEC, AFP, JCH, ADF, JTRW, MJO, MCO, PAH, JH), Division of Psychological Medicine and Clinical Neurosciences, Cardiff University, Cardiff, United Kingdom; Lieber Institute for Brain Development (LC-T, EEB, DRW, AEJ) and Centre for Computational Biology (LC-T, AEJ), Johns Hopkins University Medical Campus; Departments of Psychiatry, Neurology, Neuroscience and Genetic Medicine (DRW), Johns Hopkins School of Medicine; and the Department of Biostatistics (AEJ), Johns Hopkins Bloomberg School of Public Health, Baltimore, Maryland.

$\mathrm{AEJ}$ and $\mathrm{JH}$ contributed equally to this work.

Address correspondence to Nicholas E. Clifton, Ph.D., at cliftonne@ cardiff.ac.uk, or Andrew E. Jaffe, Ph.D., at andrew.jaffe@libd.org.

Received Oct 5, 2020; revised Feb 26, 2021; accepted Mar 4, 2021.

Supplementary material cited in this article is available online at https:// doi.org/10.1016/j.biopsych.2021.03.009.

\section{REFERENCES}

1. Cross-Disorder Group of the Psychiatric Genomics Consortium, Lee SH, Ripke S, Neale BM, Faraone SV, Purcell SM, et al. (2013): Genetic relationship between five psychiatric disorders estimated from genome-wide snps. Nat Genet 45:984-994.

2. Cardno AG, Owen MJ (2014): Genetic relationships between schizophrenia, bipolar disorder, and schizoaffective disorder. Schizophr Bull 40:504-515.

3. Owen MJ, O'Donovan MC (2017): Schizophrenia and the neurodevelopmental continuum: Evidence from genomics. World Psychiatry 16:227-235.

4. Doherty JL, Owen MJ (2014): Genomic insights into the overlap between psychiatric disorders: Implications for research and clinical practice. Genome Med 6:29.

5. Brainstorm Consortium, Anttila V, Bulik-Sullivan B, Finucane HK, Walters RK, Bras J, et al. (2018): Analysis of shared heritability in common disorders of the brain. Science 360:eaap8757.

6. Cross-Disorder Group of the Psychiatric Genomics Consortium (2019): Genomic relationships, novel loci, and pleiotropic mechanisms across eight psychiatric disorders. Cell 179:1469-1482.e11.

7. Parellada M, Gomez-Vallejo S, Burdeus M, Arango C (2017): Developmental differences between schizophrenia and bipolar disorder. Schizophr Bull 43:1176-1189.

8. Owen MJ (2014): New approaches to psychiatric diagnostic classification. Neuron 84:564-571.

9. Kessler RC, Amminger GP, Aguilar-Gaxiola S, Alonso J, Lee S, Ustün TB (2007): Age of onset of mental disorders: A review of recent literature. Curr Opin Psychiatry 20:359-364.
10. Weinberger DR (2017): Future of days past: Neurodevelopment and schizophrenia. Schizophr Bull 43:1164-1168.

11. Bimbaum R, Weinberger DR (2017): Genetic insights into the neurodevelopmental origins of schizophrenia. Nat Rev Neurosci 18:727-740.

12. Marangoni C, Hernandez M, Faedda GL (2016): The role of environmental exposures as risk factors for bipolar disorder: A systematic review of longitudinal studies. J Affect Disord 193:165-174.

13. Varese F, Smeets F, Drukker M, Lieverse R, Lataster T, Viechtbauer W, et al. (2012): Childhood adversities increase the risk of psychosis: A meta-analysis of patient-control, prospective- and cross-sectional cohort studies. Schizophr Bull 38:661-671.

14. Demjaha A, MacCabe JH, Murray RM (2012): How genes and environmental factors determine the different neurodevelopmental trajectories of schizophrenia and bipolar disorder. Schizophr Bull 38:209-214.

15. Hall J, Trent S, Thomas KL, O’Donovan MC, Owen MJ (2015): Genetic risk for schizophrenia: Convergence on synaptic pathways involved in plasticity. Biol Psychiatry 77:52-58.

16. Fromer M, Pocklington AJ, Kavanagh DH, Williams HJ, Dwyer S, Gormley P, et al. (2014): De novo mutations in schizophrenia implicate synaptic networks. Nature 506:179-184.

17. Pardiñas AF, Holmans P, Pocklington AJ, Escott-Price V, Ripke S, Carrera N, et al. (2018): Common schizophrenia alleles are enriched in mutation-intolerant genes and in regions under strong background selection. Nat Genet 50:381-389.

18. Kirov G, Pocklington AJ, Holmans P, Ivanov D, Ikeda M, Ruderfer D, et al. (2012): De novo cnv analysis implicates specific abnormalities of postsynaptic signalling complexes in the pathogenesis of schizophrenia. Mol Psychiatry 17:142-153.

19. Purcell SM, Moran JL, Fromer M, Ruderfer D, Solovieff N, Roussos P, et al. (2014): A polygenic burden of rare disruptive mutations in schizophrenia. Nature 506:185-190.

20. Howard DM, Adams MJ, Clarke TK, Hafferty JD, Gibson J, Shirali M, et al. (2019): Genome-wide meta-analysis of depression identifies 102 independent variants and highlights the importance of the prefrontal brain regions. Nat Neurosci 22:343-352.

21. Stahl EA, Breen G, Forstner AJ, McQuillin A, Ripke S, Trubetskoy V, et al. (2019): Genome-wide association study identifies 30 loci associated with bipolar disorder. Nat Genet 51:793-803.

22. Wray NR, Ripke S, Mattheisen M, Trzaskowski M, Byrne EM, Abdellaoui A, et al. (2018): Genome-wide association analyses identify 44 risk variants and refine the genetic architecture of major depression. Nat Genet 50:668-681.

23. Hammerschlag AR, de Leeuw CA, Middeldorp CM, Polderman TJC (2020): Synaptic and brain-expressed gene sets relate to the shared genetic risk across five psychiatric disorders. Psychol Med 50:1695-1705.

24. Tebbenkamp ATN, Willsey AJ, State MW, Sestan N (2014): The developmental transcriptome of the human brain: Implications for neurodevelopmental disorders. Curr Opin Neurol 27:149-156.

25. Ziats MN, Grosvenor LP, Rennert OM (2015): Functional genomics of human brain development and implications for autism spectrum disorders. Transl Psychiatry 5:e665.

26. Maurano MT, Humbert R, Rynes E, Thurman RE, Haugen E, Wang H, et al. (2012): Systematic localization of common disease-associated variation in regulatory DNA. Science 337:1190-1195.

27. Richards AL, Jones L, Moskvina V, Kirov G, Gejman PV, Levinson DF, et al. (2012): Schizophrenia susceptibility alleles are enriched for alleles that affect gene expression in adult human brain. Mol Psychiatry 17:193-201.

28. Gandal MJ, Zhang P, Hadjimichael E, Walker RL, Chen C, Liu S, et al. (2018): Transcriptome-wide isoform-level dysregulation in ASD, schizophrenia, and bipolar disorder. Science 362:eaat8127.

29. Umans BD, Battle A, Gilad Y (2021): Where are the disease-associated eQTLs? Trends Genet 37:109-124.

30. Clifton NE, Hannon E, Harwood JC, Di Florio A, Thomas KL, Holmans PA, et al. (2019): Dynamic expression of genes associated with schizophrenia and bipolar disorder across development. Trans Psychiatry 9:74.

31. Jaffe AE, Straub RE, Shin JH, Tao R, Gao Y, Collado-Torres L, et al. (2018): Developmental and genetic regulation of the human cortex transcriptome illuminate schizophrenia pathogenesis. Nat Neurosci $21: 1117-1125$ 
32. Birnbaum R, Jaffe AE, Chen Q, Hyde TM, Kleinman JE, Weinberger DR (2015): Investigation of the prenatal expression patterns of 108 schizophrenia-associated genetic loci. Biol Psychiatry 77:e43-e51.

33. Schizophrenia Working Group of the Psychiatric Genomics Consortium (2014): Biological insights from 108 schizophrenia-associated genetic loci. Nature 511:421-427.

34. Bipolar Disorder and Schizophrenia Working Group of the Psychiatric Genomics Consortium (2018): Genomic dissection of bipolar disorder and schizophrenia, including 28 subphenotypes. Cell 173:1705-1715.e16.

35. Lipska BK, Deep-Soboslay A, Weickert CS, Hyde TM, Martin CE, Herman MM, Kleinman JE (2006): Critical factors in gene expression in postmortem human brain: Focus on studies in schizophrenia. Biol Psychiatry 60:650-658.

36. Miller JA, Ding SL, Sunkin SM, Smith KA, Ng L, Szafer A, et al. (2014): Transcriptional landscape of the prenatal human brain. Nature 508:199-206.

37. Hawrylycz MJ, Lein ES, Guillozet-Bongaarts AL, Shen EH, Ng L, Miller JA, et al. (2012): An anatomically comprehensive atlas of the adult human brain transcriptome. Nature 489:391-399.

38. Andrews S (2010): FastQC: A Quality Control Tool for High Throughput Sequence Data. Cambridge, United Kingdom: Babraham Institute.

39. Bolger AM, Lohse M, Usadel B (2014): Trimmomatic: A flexible trimmer for Illumina sequence data. Bioinformatics 30:2114-2120.

40. Kim D, Langmead B, Salzberg SL (2015): HISAT: A fast spliced aligner with low memory requirements. Nat Methods 12:357-360.

41. Liao Y, Smyth GK, Shi W (2014): featureCounts: An efficient general purpose program for assigning sequence reads to genomic features. Bioinformatics 30:923-930.

42. The Gene Ontology Consortium (2017): Expansion of the gene ontology knowledgebase and resources. Nucleic Acids Res 45:D331-D338.

43. Koopmans F, van Nierop P, Andres-Alonso M, Byrnes A, Cijsouw T, Coba MP, et al. (2019): SynGO: An evidence-based, expert-curated knowledge base for the synapse. Neuron 103:217-234.e4.

44. de Leeuw CA, Mooij JM, Heskes T, Posthuma D (2015): MAGMA: Generalized gene-set analysis of GWAS data. PLoS Comput Biol 11: e1004219.

45. Network and Pathway Analysis Subgroup of Psychiatric Genomics Consortium (2015): Psychiatric genome-wide association study analyses implicate neuronal, immune and histone pathways. Nat Neurosci 18:199-209.

46. 1000 Genomes Project Consortium, Auton A, Brooks LD, Durbin RM, Garrison EP, Kang HM, et al. (2015): A global reference for human genetic variation. Nature 526:68-74.

47. Benjamini $Y$, Hochberg $Y$ (1995): Controlling the false discovery rate: A practical and powerful approach to multiple testing. J R Stat Soc B Methodol 57:289-300.

48. Ritchie ME, Phipson B, Wu D, Hu Y, Law CW, Shi W, Smyth GK (2015): limma powers differential expression analyses for RNA-sequencing and microarray studies. Nucleic Acids Res 43:e47.

49. de Leeuw CA, Stringer S, Dekkers IA, Heskes T, Posthuma D (2018): Conditional and interaction gene-set analysis reveals novel functional pathways for blood pressure. Nat Commun 9:3768.

50. Kiezun A, Garimella K, Do R, Stitziel NO, Neale BM, McLaren PJ, et al. (2012): Exome sequencing and the genetic basis of complex traits. Nat Genet 44:623-630.

51. Rees E, Carrera N, Morgan J, Hambridge K, Escott-Price V, Pocklington AJ, et al. (2019): Targeted sequencing of 10,198 samples confirms abnormalities in neuronal activity and implicates voltagegated sodium channels in schizophrenia pathogenesis. Biol Psychiatry 85:554-562.

52. Perova T, Wasserman MJ, Li PP, Warsh JJ (2008): Hyperactive intracellular calcium dynamics in B lymphoblasts from patients with bipolar I disorder. Int J Neuropsychopharmacol 11:185-196.

53. Hough C, Lu SJ, Davis CL, Chuang DM, Post RM (1999): Elevated basal and thapsigargin-stimulated intracellular calcium of platelets and lymphocytes from bipolar affective disorder patients measured by a fluorometric microassay. Biol Psychiatry 46:247-255.

54. Dubovsky SL, Christiano J, Daniell LC, Franks RD, Murphy J, Adler L, et al. (1989): Increased platelet intracellular calcium concentration in patients with bipolar affective disorders. Arch Gen Psychiatry 46:632638.

55. Harrison PJ, Hall N, Mould A, Al-Juffali N, Tunbridge EM (2019): Cellular calcium in bipolar disorder: Systematic review and metaanalysis [published online ahead of print Dec 4]. Mol Psychiatry.

56. Berridge MJ (2014): Calcium signalling and psychiatric disease: Bipolar disorder and schizophrenia. Cell Tissue Res 357:477-492.

57. Simms BA, Zamponi GW (2014): Neuronal voltage-gated calcium channels: Structure, function, and dysfunction. Neuron 82:24-45.

58. Nanou E, Catterall WA (2018): Calcium channels, synaptic plasticity, and neuropsychiatric disease. Neuron 98:466-481.

59. Zandi PP, Avramopoulos D, Willour VL, Huo Y, Miao K, Mackinnon DF, et al. (2007): SNP fine mapping of chromosome 8 q24 in bipolar disorder. Am J Med Genet B Neuropsychiatr Genet 144B:625-630.

60. Zhang P, Xiang N, Chen Y, Sliwerska E, Mclnnis MG, Burmeister M, Zöllner S (2010): Family-based association analysis to finemap bipolar linkage peak on chromosome 8q24 using 2,500 genotyped SNPs and 15,000 imputed SNPs. Bipolar Disord 12:786-792.

61. Borsotto M, Cavarec L, Bouillot M, Romey G, Macciardi F, Delaye A, et al. (2007): PP2A-Bgamma subunit and KCNQ2 K+ channels in bipolar disorder. Pharmacogenomics J 7:123-132.

62. Judy JT, Zandi PP (2013): A review of potassium channels in bipolar disorder. Front Genet 4:105.

63. Balaraman Y, Lahiri DK, Nurnberger Jl (2015): Variants in ion channel genes link phenotypic features of bipolar illness to specific neurobiological process domains. Mol Neuropsychiatry 1:23-35.

64. Jan LY, Jan YN (2012): Voltage-gated potassium channels and the diversity of electrical signalling. J Physiol 590:2591-2599.

65. Chen X, Yuan LL, Zhao C, Birnbaum SG, Frick A, Jung WE, et al. (2006): Deletion of Kv4.2 gene eliminates dendritic A-type K+ current and enhances induction of long-term potentiation in hippocampal CA1 pyramidal neurons. J Neurosci 26:12143-12151.

66. Imbrici P, Camerino DC, Tricarico D (2013): Major channels involved in neuropsychiatric disorders and therapeutic perspectives. Front Genet 4:76.

67. Sanders SJ, Murtha MT, Gupta AR, Murdoch JD, Raubeson MJ, Willsey AJ, et al. (2012): De novo mutations revealed by wholeexome sequencing are strongly associated with autism. Nature 485:237-241.

68. Satterstrom FK, Kosmicki JA, Wang J, Breen MS, De Rubeis S, An JY, et al. (2020): Large-scale exome sequencing study implicates both developmental and functional changes in the neurobiology of autism. Cell 180:568-584.e23.

69. Chateauvieux S, Morceau F, Dicato M, Diederich M (2010): Molecular and therapeutic potential and toxicity of valproic acid. J Biomed Biotechnol 2010:479364.

70. Chen RS, Deng TC, Garcia T, Sellers ZM, Best PM (2007): Calcium channel gamma subunits: A functionally diverse protein family. Cell Biochem Biophys 47:178-186.

71. Moon AL, Haan N, Wilkinson LS, Thomas KL, Hall J (2018): CACNA1C: Association with psychiatric disorders, behavior, and neurogenesis. Schizophr Bull 44:958-965.

72. Liu Y, Blackwood DH, Caesar S, de Geus EJC, Farmer A, Ferreira MAR, et al. (2011): Meta-analysis of genome-wide association data of bipolar disorder and major depressive disorder. Mol Psychiatry 16:2-4.

73. Cross-Disorder Group of the Psychiatric Genomics Consortium (2013): Identification of risk loci with shared effects on five major psychiatric disorders: A genome-wide analysis. Lancet 381:1371-1379.

74. Splawski I, Timothy KW, Sharpe LM, Decher N, Kumar P, Bloise R, et al. (2004): Ca(V)1.2 calcium channel dysfunction causes a multisystem disorder including arrhythmia and autism. Cell 119:19-31.

75. Kramer AA, Ingraham NE, Sharpe EJ, Mynlieff M (2012): Levels of $\mathrm{Ca}(\mathrm{V}) 1.2 \mathrm{~L}$-type $\mathrm{Ca}(2+)$ channels peak in the first two weeks in rat hippocampus whereas $\mathrm{Ca}(\mathrm{V}) 1.3$ channels steadily increase through development. J Signal Transduct 2012:597214.

76. Billingsley KJ, Manca M, Gianfrancesco O, Collier DA, Sharp H, Bubb VJ, Quinn JP (2018): Regulatory characterisation of the 
schizophrenia-associated CACNA1C proximal promoter and the potential role for the transcription factor EZH2 in schizophrenia aetiology. Schizophr Res 199:168-175.

77. Dedic N, Pöhlmann ML, Richter JS, Mehta D, Czamara D, Metzger MW, et al. (2018): Cross-disorder risk gene CACNA1C differentially modulates susceptibility to psychiatric disorders during development and adulthood. Mol Psychiatry 23:533-543.

78. Clark MB, Wrzesinski T, Garcia AB, Hall NAL, Kleinman JE, Hyde T, et al. (2020): Long-read sequencing reveals the complex splicing profile of the psychiatric risk gene CACNA1C in human brain. Mol Psychiatry 25:37-47.

79. Weinberger DR, Berman KF, Illowsky BP (1988): Physiological dysfunction of dorsolateral prefrontal cortex in schizophrenia. III. A new cohort and evidence for a monoaminergic mechanism. Arch Gen Psychiatry 45:609-615.

80. Selemon LD, Zecevic N (2015): Schizophrenia: A tale of two critical periods for prefrontal cortical development. Transl Psychiatry 5:e623.

81. Hare BD, Duman RS (2020): Prefrontal cortex circuits in depression and anxiety: Contribution of discrete neuronal populations and target regions. Mol Psychiatry 25:2742-2758.

82. Gogtay N, Giedd JN, Lusk L, Hayashi KM, Greenstein D, Vaituzis AC et al. (2004): Dynamic mapping of human cortical development during childhood through early adulthood. Proc Natl Acad Sci U S A 101:8174-8179.

83. Raznahan A, Lerch JP, Lee N, Greenstein D, Wallace GL, Stockman M, et al. (2011): Patterns of coordinated anatomical change in human cortical development: A longitudinal neuroimaging study of maturational coupling. Neuron 72:873-884.

84. Nowakowski TJ, Bhaduri A, Pollen AA, Alvarado B, Mostajo-Radji MA, Di Lullo E, et al. (2017): Spatiotemporal gene expression trajectories reveal developmental hierarchies of the human cortex. Science 358:1318-1323.

85. Fan X, Fu Y, Zhou X, Sun L, Yang M, Wang M, et al. (2020): Singlecell transcriptome analysis reveals cell lineage specification in temporal-spatial patterns in human cortical development. Sci Adv 6: eaaz2978.

86. Lencz T, Malhotra AK (2015): Targeting the schizophrenia genome: A fast track strategy from GWAS to clinic. Mol Psychiatry 20:820826.

87. Weiss N, Zamponi GW (2019): T-type channel druggability at a crossroads. ACS Chem Neurosci 10:1124-1126.

88. Zhao C, Hong L, Riahi S, Galpin JD, Ahern CA, Tobias DJ, Tombola F (2020): Rationally designed proton channel inhibitors reveal a druggable pocket in a voltage-sensing domain. Biophys J 118:111a. 